\title{
Safety and occlusion rates of surgical treatment of unruptured intracranial aneurysms: a systematic review and meta-analysis of the literature from 1990 to 2011
}

\author{
Marc Kotowski, ${ }^{1,2}$ Olivier Naggara, ${ }^{3}$ Tim E Darsaut, ${ }^{1,4,5}$ Suzanne Nolet, ${ }^{5}$ \\ Guylaine Gevry, ${ }^{5}$ Evgueni Kouznetsov, ${ }^{1}$ Jean Raymond ${ }^{1,5}$
}

- Additional supplementary files are published online only. To view these files please visit the journal online (http://dx.doi. org/10.1136/jnnp-2011302068).

${ }^{1}$ Department of Radiology, Centre hospitalier de I'Université de Montréal (CHUM), Notre-Dame Hospital, Montreal, Canada

${ }^{2}$ Department of Neurosurgery, Centre hospitalier universitaire Vaudois, Lausanne, Switzerland ${ }^{3}$ Département d'Imagerie Morphologique and

Fonctionnelle, Université Paris Descartes, Sorbonne Paris Cité, INSERM UMR 894, Hôpital Sainte-Anne, Paris, France ${ }^{4}$ Mackenzie Health Sciences Centre, Division of Neurosurgery, Department of Surgery, University of Alberta Hospital, Edmonton, Alberta, Canada

${ }^{5} \mathrm{CHUM}$ Research Centre, Interventional Neuroradiology Laboratory, Notre-Dame Hospital, Montreal, Canada

Correspondence to Dr J Raymond, CHUM NotreDame Hospital, Department of Radiology, 1560 Sherbrooke East, Pav Simard, Z12909, Montreal, Canada H2L 4M1; jean.raymond@umontreal.ca

Received 19 December 2011 Revised 5 June 2012 Accepted 22 August 2012 Published Online First 25 September 2012

- http://dx.doi.org/10.1136/ jnnp-2012-303133

\section{ABSTRACT}

Background and purpose Surgical clipping of unruptured intracranial aneurysms (UIAs) has recently been challenged by the emergence of endovascular treatment. We performed an updated systematic review and meta-analysis on the surgical treatment of UIAs, in an attempt to determine the aneurysm occlusion rates and safety of surgery in the modern era.

Methods A detailed protocol was developed prior to conducting the review according to the Cochrane Collaboration guidelines. Electronic databases spanning January 1990-April 2011 were searched, complemented by hand searching. Heterogeneity was assessed using $\mathrm{l}^{2}$, and publication bias with funnel plots. Surgical mortality and morbidity were analysed with weighted random effect models.

Results 60 studies with 9845 patients harbouring 10845 aneurysms were included. Mortality occurred in 157 patients $\left(1.7 \% ; 99 \%\right.$ Cl $0.9 \%$ to $\left.3.0 \% ; I^{2}=82 \%\right)$. Unfavourable outcomes, including death, occurred in 692 patients $16.7 \%$; $99 \% \mathrm{Cl} 4.9 \%$ to $\left.9.0 \% ;\left.\right|^{2}=85 \%\right)$. Morbidity rates were significantly greater in higher quality studies, and with large or posterior circulation aneurysms. Reported morbidity rates decreased over time. Studies were generally of poor quality; funnel plots showed heterogeneous results and publication bias, and data on aneurysm occlusion rates were scant. Conclusions In studies published between 1990 and 2011, clipping of UIAs was associated with $1.7 \%$ mortality and $6.7 \%$ overall morbidity. The reputed durability of clipping has not been rigorously documented. Due to the quality of the included studies, the available literature cannot properly guide clinical decisions.

\section{INTRODUCTION}

Unruptured intracranial aneurysms (UIAs) are increasingly detected and treated ${ }^{1}{ }^{2}$ but the best management remains uncertain. ${ }^{3}$ Surgical clipping, for years considered the gold standard, has been challenged by the emergence of endovascular treatment (EVT), a minimally invasive alternative. ${ }^{145}$ Successful clipping is reputed to result in definitive aneurysm exclusion, with retreatment rates as low as $1.5 \%{ }^{6}$ Proponents of EVT claim lower treatment related risks, ${ }^{7-10}$ perhaps at the cost of lower long term occlusion rates, ${ }^{11}$ but a valid, randomised comparison has never been performed. ${ }^{12}$

A prevailing view is that clinical decision making 'requires knowledge of the natural history of UIAs and of the risks of repairing them ${ }^{13}$ and 'adequately balancing benefits and risks requires reliable data on surgical outcome', ${ }^{14}$ although this management philosophy has been questioned. ${ }^{15}$ Better knowledge of the risks involved in the surgical repair of UIAs may nonetheless help with counselling patients as well as provide hypotheses for clinical trials. ${ }^{12}$ The last meta-analysis of surgical outcomes following clipping of UIAs reviewed the published literature up to June $1996 .{ }^{14}$ After the publication of the ISUIA registry in 1998, those patients selected for surgery may have changed, with different clinical outcomes following clipping in the more modern era. ${ }^{13}{ }^{16}$ We conducted an updated review, aiming to: (a) assess morbidity and case fatality rates following surgical clipping of UIAs; (b) identify patient or aneurysm characteristics associated with greater surgical risks; and (c) determine occlusion rates following surgical clipping, according to published clinical or angiographic results.

\section{MATERIAL AND METHODS}

A detailed protocol was developed prior to conducting this review according to guidelines published by the Cochrane Collaboration, ${ }^{17}$ the Meta-Analysis of Observational Studies in Epidemiology criteria ${ }^{18}$ and the Preferred Reporting Items for Systematic Reviews and Meta-Analyses statement, ${ }^{19}$ including methods for literature search, publication eligibility, assessment of quality of reporting, data extraction and analysis.

\section{Search strategy}

The literature in French and English on surgical treatment of UIAs was reviewed from 1990 to 2011, using PubMed (Medline), Embase, ISI Web of Knowledge and the Cochrane Library, complemented by hand searching and extensive cross referencing. Only those studies containing outcome data were included for full text review (see supplementary data available online only). In the event of multiple reports with data on the same patients, only the most recent was included. In studies where only a subset of patients met the inclusion criteria, these were selectively included. Multiple aneurysm patients treated for an UIA with a remote history of SAH from a different lesion were included. 


\section{Selection criteria}

The prespecified inclusion criteria were: (1) explicitly reported mortality or permanent morbidity rate using a validated outcome scale $20-22$ or individual data allowing recalculation into crude numbers. Population based studies based on ICD-10 discharge databases were thus excluded ${ }^{23}$; (2) saccular, nondissecting, non-infectious, previously untreated UIAs not associated with arteriovenous malformations; (3) report contained a minimum of 10 patients with UIAs; (4) peer reviewed original study; and (5) score $>6$ on the Strengthening the Reporting of Observational Studies in Epidemiology (STROBE) scale. $^{2425}$

\section{Data extraction}

Data were extracted by one author (MK) using a standardised critical appraisal and digitised data extraction form. Two authors (TED and EK) reviewed a random sample of $40 \%$ of the studies and extracted data. Disagreements were resolved by consensus after reviewing studies.

\section{Study characteristics}

Data included type of study, single or multicentre, retro- or prospective data collection, consecutive or non-consecutive patient accrual, year, journal, language, country of origin of publication and study interval. Quality of reporting was assessed for all included publications using the STROBE 22 point checklist. We added 1 point for each of the following criteria: (a) randomised controlled trial (RCT), (b) independent clinical outcome assessment, (c) prospective study design, (d) consecutive patient inclusion and (e) multicentre study. Study scores were classified as poor (6-12), average (13-18) or good (19-27).

\section{Patient and aneurysm characteristics}

Data recorded included the number of patients, mean age, sex, clinical presentation and number of patients with multiple aneurysms. Data on aneurysms characteristics included number, location and size of aneurysms. Additional information on treatment modalities (eg, clipping, wrapping, trapping or bypass procedures) was extracted where possible.

\section{Outcomes}

The safety endpoints extracted were: (a) death and (b) disability, defined as either a modified Rankin Scale ${ }^{21}$ score of $>2$, a Glasgow Outcome Scale ${ }^{20}$ score of $<4$ or a EGFP (Excellent, Good, Fair, Poor) Scale ${ }^{22}$ score of 'fair' and 'poor'. In patients with a prior deficit, a loss of 1 point on any outcome scale was considered an unfavourable outcome. When poor outcomes from the treatment of unruptured aneurysms were not distinguished from poor outcomes antedating surgery in populations that could have included patients with a previous history of subarachnoid haemorrhage from another aneurysm, we attributed such outcomes to surgery. Patient populations with only mean outcome or mean difference pre- and post-intervention outcome scores reported were excluded. When the author described a deficit without specifying the outcome, we attributed a modified Rankin Scale or EGFP score from the description.

The time of follow-up clinical assessment was classified as (1) short term (inhospital morbidity or within 1 month after intervention), (2) mid term (1 month to 1 year after intervention) or (3) long term (>1 year after intervention). When time of assessment was unspecified, outcome data were considered short term.

\section{Occlusion rate}

Data on aneurysm occlusion included number of patients with postoperative imaging, and type and timing of imaging, with results categorised as complete or incomplete occlusion. Incomplete occlusion was defined as attempted yet unsuccessful complete obliteration of the aneurysm dome, including cases where a residual neck remained. Efficacy regarding the prevention of SAH at long term follow-up was also sought in terms of bleeding rates, as well as retreatment rates.

\section{Subgroups}

Clinical outcome data were examined using predefined subgroups: (1) age dichotomised as $\leq$ or $>55$ years; (2) anterior versus posterior circulation location; and (3) aneurysm size, categorised as small $(<10 \mathrm{~mm})$, large $(10-24 \mathrm{~mm})$ or giant $(\geq 25 \mathrm{~mm}$ ). Other predefined subgroups included the publication quality score, dichotomised as high $(19-26)$ or low $(<19)$, origin of the study, categorised as North American, European or Australasian, and era of publication year, dichotomised to 1990-2000 or 2001-2011.

Manuscripts used for subgroup analyses are detailed in the supplementary file 2 (available online only).

\section{Analyses}

The degree of heterogeneity between studies was reported using $\mathrm{I}^{2}=((\mathrm{Q}-\mathrm{df}) / \mathrm{Q}) \times 100 \%$, where $\mathrm{Q}$ is the $\chi^{2}$ statistic and $\mathrm{df}$ is the number of degrees of freedom. ${ }^{26} 27 \mathrm{I}^{2}$ describes the per cent variability in effect estimates due to heterogeneity rather than sampling error. Heterogeneity was classified as moderate $\left(\mathrm{I}^{2} \geq 30 \%\right)$, substantial $\left(\mathrm{I}^{2} \geq 50 \%\right)$ or considerable $\left(\mathrm{I}^{2} \geq 75 \%\right)$. Due to the degree of heterogeneity found, we used a DerSimonianLaird random effect weight (REW) model for all analyses. Corresponding 99\% CI for single proportions were determined using the binomial method. We assessed publication bias with scatterplots according to study size or precision. ${ }^{28} 29 \mathrm{SE}$ was chosen for the vertical axis and the logit event rate for the horizontal axis, defined as $\operatorname{logit}(p)=\log (p)-\log (1-p)$, where $p$ is the event rate. ${ }^{30}$ All analyses were performed using Comprehensive MetaAnalysis 2.0 (Biostat Inc, Englewood, New Jersey, USA) and Graphpad QuickCalcs (GraphPad Inc, La Jolla, California, USA).

\section{RESULTS}

\section{Studies}

Study selection is summarised in figure 1. The initial search yielded 6529 articles. Full text review of 595 articles was performed after selection criteria were met, or when uncertainty remained after review of the title and abstract. Studies were excluded $(n=535)$ most commonly when clinical outcomes were not differentiated for ruptured compared with unruptured aneurysms (eliminating 105 studies reporting 10759 patients).

Table 1 summarises the study characteristics; the 60 included studies reported outcomes for 9845 patients with 10845 unruptured aneurysms. The median quality score of included publications was $8 \pm 2.9$ (range 6-19). Reporting was graded 'good' for one (1.6\%), 'average' for eight (13.3\%) and 'poor' for 51 (85\%) studies. One RCT was identified ${ }^{31} ; 59$ (98.3\%) studies were observational, 11 (18.3\%) prospective, nine (15\%) consecutive and $11(18.3 \%)$ multicentre studies. Three (5\%) studies had independent outcome assessment. Twenty-four (40\%) 


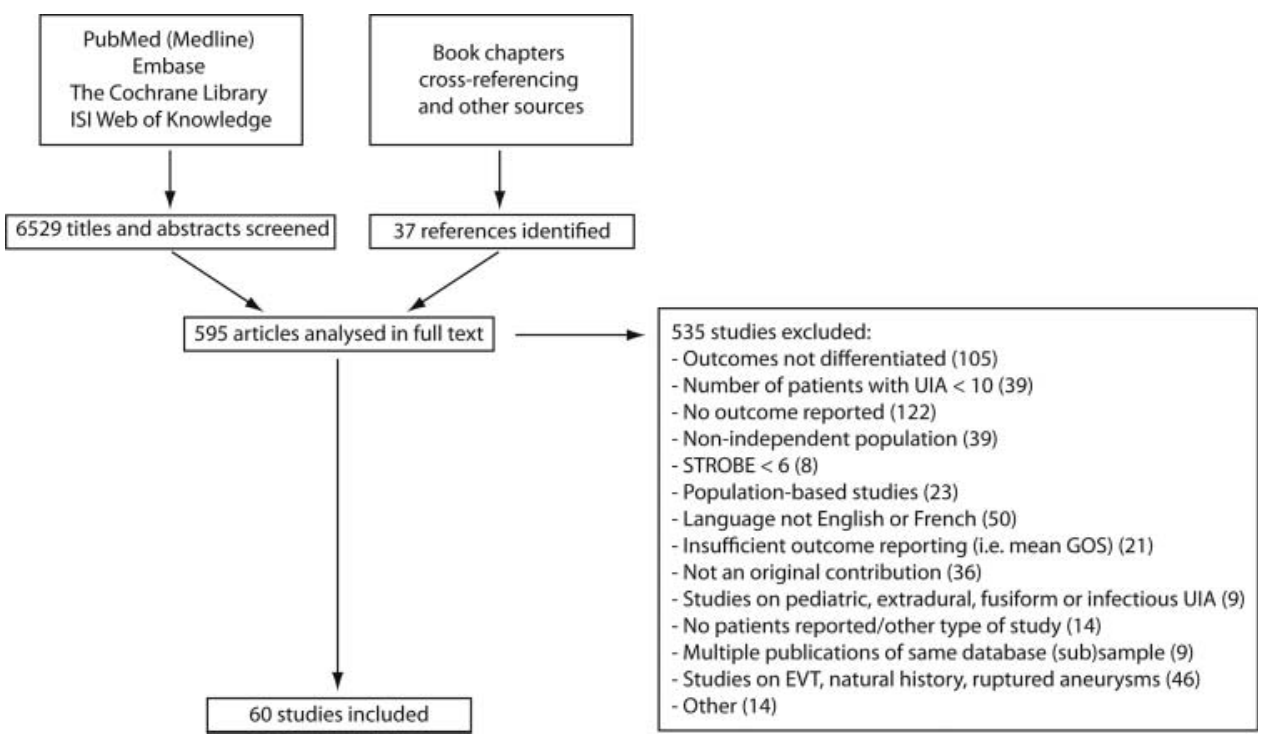

Figure 1 Flowchart of the literature search method. EVT, endovascular treatment; GOS, Glasgow Outcome Scale; UIA, unruptured intracranial aneurysm.

studies comprising 4901 (49.8\%) patients were of North American origin, 17 (28.3\%) studies comprising $1351(13.7 \%)$ were conducted in Europe and $18(30 \%)$ studies with 3583 (36.4\%) patients were from Australasia, including Japan. One additional study reported on 10 (0.1\%) South American patients.

\section{Table 1 Publication characteristics}

\begin{tabular}{|c|c|c|}
\hline & Studies & $\%$ \\
\hline \multicolumn{3}{|l|}{ Quality score } \\
\hline 6-12 (poor) & 51 & 85 \\
\hline 13-18 (average) & 8 & 13 \\
\hline 19-26 (good) & 1 & 2 \\
\hline Median $( \pm S D)$ & $8( \pm 2.9)$ & \\
\hline Range & $6-22$ & \\
\hline \multicolumn{3}{|l|}{ Patient enrolment } \\
\hline Prospective & 11 & 18 \\
\hline Retrospective & 49 & 82 \\
\hline Consecutive & 9 & 15 \\
\hline Non-consecutive & 51 & 85 \\
\hline \multicolumn{3}{|l|}{ Study design } \\
\hline Multicentre & 11 & 18 \\
\hline Single centre & 49 & 82 \\
\hline \multicolumn{3}{|l|}{ Type of study } \\
\hline Randomised controlled trial & 1 & 1.7 \\
\hline Observational studies & 59 & 98 \\
\hline \multicolumn{3}{|l|}{ Outcome assessment } \\
\hline Independent clinical outcome assessment & 3 & 5 \\
\hline Modified Rankin Scale & 9 & 15 \\
\hline Glasgow Outcome Scale & 33 & 55 \\
\hline EGFP & 20 & 33 \\
\hline Loss of follow-up reported & 11 & 18 \\
\hline \multicolumn{3}{|l|}{ Time of outcome assessment } \\
\hline Short term ('discharge' to 1 month) & 11 & 18 \\
\hline Mid term (>1 month to 1 year postop) & 23 & 38 \\
\hline Long term (>1 year postop) & 3 & 5 \\
\hline Patient years' follow-up & 11 & 18 \\
\hline No information on time of outcome measurement & 12 & 20 \\
\hline
\end{tabular}

\section{Patients}

Patient characteristics are summarised in table 2. Reported clinical outcomes were short term for 5984 (60.8\%), mid term for $3013(30.6 \%)$ and long term for 152 (1.5\%) patients. In 696 (7.1\%) cases, outcome was reported using the patient years method.

Details on type of surgical treatment were specified in $26(43.3 \%)$ publications for 3437 (34.9\%) patients. Details on periprocedural complications were provided in $18(30 \%)$ publications.

Table 2 Patient characteristics

\begin{tabular}{|c|c|c|}
\hline & Patients & $\%$ \\
\hline Total No of patients & 9845 & 100 \\
\hline Female & 5427 & 55.1 \\
\hline Male & 2390 & 24.3 \\
\hline Sex not reported & 2028 & 20.6 \\
\hline $\mathrm{M} / \mathrm{F}$ ratio & 0.44 & \\
\hline Age range (years) & $1-86$ & \\
\hline Patients with multiple aneurysms & 1263 & 12.8 \\
\hline \multicolumn{3}{|l|}{ Presentation } \\
\hline Symptomatic & 1028 & 10.4 \\
\hline Asymptomatic & 2342 & 23.8 \\
\hline Cranial nerve palsy & 312 & 3.2 \\
\hline Previous episode of SAH from another lesion & 628 & 6.4 \\
\hline Not reported & 5535 & 56.2 \\
\hline \multicolumn{3}{|l|}{ Treatment } \\
\hline Clipping & 3122 & 31.7 \\
\hline Clipping + bypass & 28 & 0.3 \\
\hline Wrapping & 118 & 1.2 \\
\hline Wrapping+bypass & 3 & 0.0 \\
\hline Clipping + wrapping & 13 & 0.1 \\
\hline Proximal occlusion+bypass & 53 & 0.5 \\
\hline Trapping & 54 & 0.5 \\
\hline Bipolar coagulation and gauze reinforcement & 39 & 0.4 \\
\hline Proximal occlusion & 120 & 1.2 \\
\hline Not reported & 6295 & 63.9 \\
\hline
\end{tabular}


Table 3 Aneurysm characteristics

\begin{tabular}{lcc}
\hline & No of aneurysms & $\%$ \\
\hline Total No of aneurysms & 10845 & 100 \\
Location of aneurysms & & \\
$\quad$ Anterior circulation aneurysms & 6766 & 62.4 \\
ICA & 2732 & 25.2 \\
MCA & 2552 & 23.5 \\
$\quad$ ACA & 1205 & 11.1 \\
Posterior circulation aneurysms & 1014 & 9.3 \\
Basilar tip & 318 & 2.9 \\
All others & 243 & 2.2 \\
Location not reported & 3065 & 28.3 \\
Size (mm) & & \\
$<10$ & 3771 & 34.8 \\
10-24 & 1704 & 15.7 \\
$\geq 25$ & 476 & 4.4 \\
$\quad$ Not reported & 4894 & 45.1 \\
Other aneurysm characteristics & & \\
$\quad$ Thrombosed & 76 & 0.7 \\
Calcified & 30 & 0.3 \\
\hline *Regrouped size: aneurysms 7-12 mm were classified as small, 6-16 mm as large.
\end{tabular}

$\mathrm{ACA}$, anterior cerebral artery; ICA, internal carotid artery; MCA, middle cerebral artery.

\section{Aneurysms}

Location was specified in $44(73.3 \%)$ studies for $7780(71.7 \%)$ aneurysms: 6766 (87\%) were located in the anterior and 1014 $(13 \%)$ in the posterior circulation. Aneurysm characteristics are summarised in table 3 .

\section{Clinical outcomes}

In total, 157 of 9845 patients died after surgery (REW average: $1.7 \% ; \quad 99 \%$ CI $0.9 \%$ to $\left.3.0 \% ; \quad I^{2}=82.2 \% ; \quad p_{(\text {het })}<0.001\right)$. Unfavourable outcome, including death, up to 1 year after surgery, was found in 692 patients (REW average: 6.7\%; 99\% CI $4.9 \%$ to $9.0 \%$; $\mathrm{I}^{2}=85.1 \%$; $\left.\mathrm{p}_{\text {(het) }}<0.001\right)$.

Funnel plot analysis (figure 2) showed substantial bias, with data points concentrated in the lower left hand corner of the plot, which was more pronounced for the estimate of morbidity than mortality. Among studies with a small SE, three reported higher event rates than others: two studies ${ }^{32} 33$ reported results concerning treatment of posterior circulation aneurysms, and the other large and giant anterior circulation aneurysms. $^{34}$ Two studies ${ }^{35} 36$ reported death rates that were not within the lower limit of the CI; one was a national survey and the other was a stratification of outcome in terms of hospital caseload and operations of individual surgeons.

\section{Subgroup meta-analysis of surgical risk factors}

Table 4 summarises the results for the predefined subgroups. Comparative outcomes with respect to patient age were reported for 500 (5.1\%) patients, with respect to aneurysm location for 3010 (30.6\%) patients and for aneurysm size for $591(6 \%)$ patients.

Aneurysm location and size reached statistical significance in terms of surgical risk factors $(p<0.0001)$. The relative risk for unfavourable outcome of patients with posterior circulation aneurysms was 4.1 (99\% CI 2.3 to 7.6$)$ and the relative risk for patients with large and giant aneurysms was 3.5 (99\% CI 1.4 to 8.9). Analysis of age as a risk factor did not reach significance (figure 3).

\section{Subgroup meta-analysis of the literature}

High quality studies (19-27 points) reported a significantly higher rate of unfavourable outcomes, including mortality, compared with poor and average quality studies $(p<0.0001)$. Most high quality studies $(67 \%)$ were conducted in North America. More recent studies (2001-2011) tended to report fewer unfavourable outcomes compared with studies from 1990 to 2000 ( $p<0.0001)$. Funnel plots demonstrated persistent bias in both eras. Reported clinical outcomes were significantly different depending on origin of publication, with North American studies publishing higher rates of unfavourable outcomes $(p<0.0001)$

\section{Occlusion rates}

The majority of publications did not report aneurysm occlusion rates. Data were missing for $82.2 \%$ of all clipped aneurysms. Information on occlusion rates was available for $24(40 \%)$ of the included publications. Of those, five stated that all aneurysms were completely occluded without detailing how results were verified. Only nine (15\%) studies defined complete versus
Figure 2 Funnel plot. (A) Death rate and $(B)$ morbidity and death rates. Individual points correspond to published rates from individual studies. The wide distribution and asymmetry suggest bias. Studies with a larger SE tended to report lower morbidity and death rates.

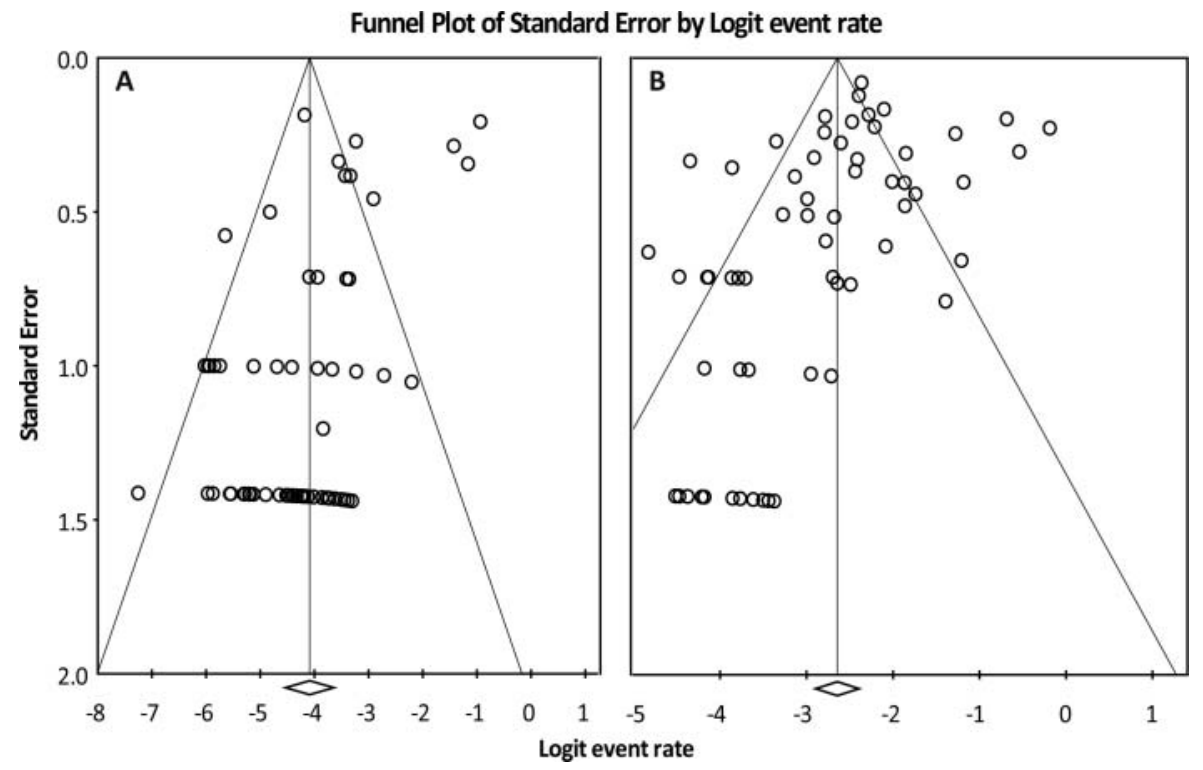


Table 4 Subgroup meta-analysis

\begin{tabular}{|c|c|c|c|c|}
\hline & Unfavourable outcomet (\%) (99\% Cl) & Heterogeneity $\mathbf{0}$ value $/ \mathbf{l}^{2}$ & p Value (het)* & p Value (sig) \\
\hline \multicolumn{5}{|l|}{ Quality of study } \\
\hline High quality studies ( $n=9 \neq, 2704$ patients, 3027 aneurysms) & $9.9(4.9 \%$ to $19.0 \%)$ & $68.1 / 88.3 \%$ & $<0.0001$ & \multirow[t]{2}{*}{$<0.0001$} \\
\hline Low quality studies ( $n=51,7141$ patients, 8012 aneurysms) & $6.1(4.2 \%$ to $8.7 \%)$ & $323.2 / 84.5 \%$ & $<0.0001$ & \\
\hline \multicolumn{5}{|l|}{ Origin of study } \\
\hline North America ( $n=24,4901$ patients, 5343 aneurysms) & $9.4(5.9 \%$ to $14.6 \%)$ & $223.8 / 89.7 \%$ & $<0.0001$ & \multirow[t]{3}{*}{$<0.0001$} \\
\hline Europe ( $\mathrm{n}=17,1351$ patients, 1764 aneurysms) & $4.5(2.4 \%$ to $8.4 \%)$ & $38.7 / 58.7 \%$ & 0.001 & \\
\hline Asia ( $n=18,3583$ patients, 3920 aneurysms) & $5.2(3.1 \%$ to $8.6 \%)$ & $94.2 / 90.0 \%$ & $<0.0001$ & \\
\hline \multicolumn{5}{|l|}{ Midyear of treatment } \\
\hline 1990-2000 ( $n=22,1711$ patients, 1853 aneurysms) & $8.0(4.7 \%$ to $13.4 \%)$ & $99.1 / 78.8 \%$ & $<0.0001$ & \multirow[t]{2}{*}{$<0.0001$} \\
\hline $2001-2011$ ( $n=38,8134$ patients, 9186 aneurysms) & $6.0(4.1 \%$ to $8.7 \%)$ & $282.3 / 86.9 \%$ & $<0.0001$ & \\
\hline \multicolumn{5}{|l|}{ Age (years) } \\
\hline Mean age $>55$ ( $n=9$, 433 patients) & $11.8(6.8 \%$ to $19.8 \%)$ & $19.8 / 64.7 \%$ & 0.006 & \multirow[t]{2}{*}{0.18} \\
\hline Mean age $\leq 55$ ( $n=5,93$ patients) & $8.2(3.1 \%$ to $20.0 \%)$ & $1.7 / 0 \%$ & 0.79 & \\
\hline \multicolumn{5}{|l|}{ Location } \\
\hline Anterior ( $n=17,2461$ patients) & $5.7(2.3 \%$ to $13.3 \%)$ & $151.9 / 90.1 \%$ & $<0.001$ & \multirow[t]{2}{*}{$<0.0001$} \\
\hline Posterior ( $n=13,549$ patients) & $15.6(7.4 \%$ to $30.1 \%)$ & $52.3 / 80.9 \%$ & $<0.001$ & \\
\hline \multicolumn{5}{|l|}{ Fundus size (mm) } \\
\hline$<10$ (n=6, 240 patients) & $4.0(1.7 \%$ to $8.8 \%)$ & $0.2 / 0 \%$ & 1 & \multirow[t]{3}{*}{$<0.0001$} \\
\hline $10-25$ ( $n=7,195$ patients) & $12.1(7.0 \%$ to $19.9 \%)$ & $4.9 / 0 \%$ & 0.6 & \\
\hline$>25$ ( $n=7,156$ patients) & $26.5(8.5 \%$ to $58.3 \%)$ & $19.9 / 74.9 \%$ & 0.001 & \\
\hline
\end{tabular}

incomplete occlusion. Four (6.6\%) publications provided the timing of postoperative imaging. Recalculation from proportions into crude numbers was necessary with 10 studies. No publication provided details of adjudication of results, or discussed interobserver agreement. There were no data on long term imaging follow-up. The remaining 19 (32\%) publications (1793 patients; 2180 aneurysms) reported imaging data for 1969 postoperative examinations; $91.8 \%$ (99\% CI $90 \%$ to $93.2 \%$ ) of aneurysms were reported as completely occluded. Neck remnants were present in 3.9\% (99\% CI $2.9 \%$ to $5.2 \%$ ), with incomplete occlusions in $4.3 \%$ (99\% CI $3.3 \%$ to $5.7 \%)$.

Data on bleeding rates after surgical treatment were provided in nine $(15 \%)$ publications with $773(7.9 \%)$ patients. Three events $(0.38 \%)$ were reported during an average follow-up of 1.2 years per patient. Due to insufficient power, reliable analysis was not possible for bleeding or retreatment rates.

\section{DISCUSSION}

This review found that surgical clipping of UIAs is associated with a risk of unfavourable outcome of $6.7 \%(99 \%$ CI $4.9 \%$ to $9.0 \%$ ), and a risk of death of $1.7 \%$ ( $99 \%$ CI $0.9 \%$ to $3.0 \%$ ). However, we also found that the literature on surgical treatment of UIAs is heterogeneous, not of high quality and contains evidence of bias. Published studies often had shortcomings in design, conduct and reporting. Clinical outcomes were most commonly self-assessed, and duration of follow-up short and often incomplete. Finally, there were no reliable data on occlusion rates following clipping. We do not recommend using the results of surgical clipping obtained from this literature to formulate treatment plans.

Two previous meta-analyses have been published on morbidity and mortality of surgery for UIA. In 1994, King et al ${ }^{37}$ found a morbidity rate of $4.1 \%$ and a death rate of $1.0 \%$, whereas Raaymakers et al ${ }^{14}$ in 1998 found nearly double rates of $10.9 \%$ and $2.0 \%$, respectively. Both meta-analyses examined literature published from 1966 to 1992; different study designs and definitions were the most likely explanations for the divergent results. Raaymakers et $a l^{14}$ defined surgical morbidity as "all permanent morbidity not present before operation and ....any outcome except the most favourable...". They also reported that "half of the patients with surgical morbidity became dependent in daily life". By requiring dependence to define morbidity, approximately half of the morbidity rate of Raaymakers et al can be expected, which would give a result similar to our findings.

Clipping has been challenged by the less invasive, presumably less morbid, endovascular alternative, and recent epidemiological studies have claimed that patients with UIAs are more often discharged to long term facilities when treated with clipping than with coiling. ${ }^{23}$ While these results support the fact that patient recover more quickly from less invasive treatments, they do not prove that the risks of significant neurological morbidity are less. Epidemiological results cannot be used to judge the relative merits of each intervention as patients cannot be compared: patients with more difficult aneurysms in any location may have been referred to surgery while those with posterior circulation aneurysms may have been referred to EVT. It
Figure 3 Meta-analysis of risk factors for an unfavourable outcome. Meta-analysis of studies with dichotomised subgroups.
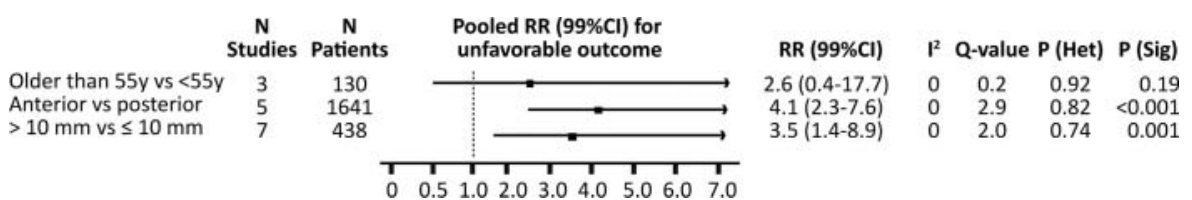
remains nevertheless tempting to compare the results of this study with a recent meta-analysis carried out by our group on the treatment related risks of EVT for UIAs. ${ }^{10}$ Analysing the literature from 2003 to 2008, we found a procedural unfavourable outcome, including mortality, of 4.8\% (REW average: $99 \%$ CI $3.4 \%$ to $5.6 \%$ ) and a mortality of $1.9 \%$ (REW average: $99 \%$ CI $1.3 \%$ to $2.6 \%$ ) with EVT of UIAs, which is comparable with the results of surgical clipping found here. However, in the absence of randomisation, any attempts to directly compare treatment outcomes remain invalid, as differences in morbidity and mortality will certainly reflect selection bias.

Surgical clipping is reputed to yield more durable aneurysm occlusion than coiling, a notion supported by a randomised study that found higher rebleeding rates after coiling than clipping of ruptured aneurysms. ${ }^{38}$ Rigorously speaking, this notion should not be extrapolated to UIAs. Occlusion rates following EVT are routinely available, and even though long term angiographic follow-up studies are infrequently done, recurrences have been documented in $24 \%$ of cases, with retreatment in at least $9 \% .{ }^{10}$ Unfortunately, the articles included in our review cannot support the superiority of clipping in terms of long term occlusion rates. Clipping is not always successful or complete, and new aneurysms may develop after surgery. ${ }^{39}$

Our results suggest that treatment risks have decreased in recent years, but it is unclear if this reflects improved surgical techniques. Changing treatment trends, shifting clinical indications and the progressive use of EVT over time are likely to have had a major influence on clinical outcomes. ${ }^{40}$

Meta-analyses usually focus on RCT results. They can be considered helpful in non-randomised studies when the literature is sufficiently homogeneous in terms of participants, interventions and outcomes to provide a meaningful summary. They may also help identify hypotheses for clinical trials. Given the heterogeneity and poor quality of the studies that were included, we want to ensure that we do not give credibility to potentially misleading results. All potential sources of biases affecting our results cannot be quantified.

There are additional limitations to our study. First, some reports may have been missed, as we included only studies in English and French, potentially introducing language bias. ${ }^{41} 42$ Second, our policy was to extract maximum information. One could argue that we should have restricted inclusion to high quality articles but this would have led to exclusion of 51 (85\%) studies. Third, we chose to restrict this review to articles published since 1990. Our justification in excluding older articles was incomparable clinical settings, technological advances, changing range of indications for surgical treatment of UIA and a literature that becomes historically less comparable with current practice. Including more patients would have increased the power of the study but perhaps at the cost of decreased generalisability of results. Finally, lack of individual patient data precluded multivariate analyses; hence we could not correct for potential confounders. Our results should be considered strictly exploratory, and caution is advised regarding use of these data for clinical decision making. We are aware that results may be used in cost effectiveness analyses, but in our view these numbers are too uncertain to use in any decisional treatment algorithm for patients with UIA.

Two dilemmas continue to plague the clinical management of patients with UIAs: whether patients eligible for both treatment options should be treated with clipping or with coiling, and whether risky preventive treatments are justified at all. ${ }^{43}$ Unfortunately, this review cannot provide answers to these fundamental questions.

\section{CONCLUSIONS}

A systematic review of the literature on surgical treatment of UIA showed substantial heterogeneity and suboptimal reporting. Surgical risks were in the range of $6-7 \%$. Reliable data on occlusion rates of surgical treatment were absent. The best management of patients with UIA remains unknown. Randomised evidence should be a priority issue in clinical neurovascular research.

Contributors MK, TED and EK reviewed the literature. MK and ON analysed the data. MK, ON, TD and JR drafted the article. SN and GG designed the extraction database. All authors reviewed the manuscript.

Competing interests None.

Provenance and peer review Not commissioned; externally peer reviewed.

\section{REFERENCES}

1. Huang MC, Baaj AA, Downes K, et al. Paradoxical trends in the management of unruptured cerebral aneurysms in the United States: analysis of nationwide database over a 10-year period. Stroke 2011;42:1730-5.

2. Vernooij MW, Ikram MA, Tanghe HL, et al. Incidental findings on brain MRI in the general population. N Engl J Med 2007;357:1821-8.

3. Raymond J. Incidental intracranial aneurysms: rationale for treatment. Curr Opin Neurol 2009;22:96-102.

4. Brinjikji W, Rabinstein AA, Kallmes DF, et al. Patient outcomes with endovascular embolectomy therapy for acute ischemic stroke: a study of the national inpatient sample: 2006 to 2008. Stroke 2011;42:1648-52.

5. Brinjikji W, Rabinstein AA, Lanzino G, et al. Patient outcomes are better for unruptured cerebral aneurysms treated at centers that preferentially treat with endovascular coiling: a study of the National Inpatient Sample 2001-2007. AJNR Am J Neuroradiol 2011;32:1065-70.

6. Raja PV, Huang J, Germanwala AV, et al. Microsurgical clipping and endovascular coiling of intracranial aneurysms: a critical review of the literature. Neurosurgery 2008;62:1187-202.

7. Johnston SC, Wilson CB, Halbach W, et al. Endovascular and surgical treatment of unruptured cerebral aneurysms: comparison of risks. Ann Neurol 2000;48:11-9.

8. Pierot L, Spelle L, Vitry F. Immediate clinical outcome of patients harboring unruptured intracranial aneurysms treated by endovascular approach: results of the ATENA study. Stroke 2008:39:2497-504

9. Lanterna LA, Tredici G, Dimitrov BD, et al. Treatment of unruptured cerebral aneurysms by embolization with Guglielmi detachable coils: case-fatality, morbidity, and effectiveness in preventing bleeding - a systematic review of the literature. Neurosurgery 2004;55:767-75.

10. Naggara $\mathbf{O N}$, White PM, Guilbert F, et al. Endovascular treatment of intracranial unruptured aneurysms: systematic review and meta-analysis of the literature on safety and efficacy. Radiology 2010;256:887-97.

11. Raymond J, Guilbert F, Weill A, et al. Long-term angiographic recurrences after selective endovascular treatment of aneurysms with detachable coils. Stroke 2003;34:1398-403.

12. Darsaut TE, Findlay JM, Raymond J. The design of the Canadian unruptured endovascular versus surgery (CURES) trial. Can J Neurol Sci 2011;38:236-41.

13. Wiebers D. Unruptured intracranial aneurysms - risk of rupture and risks of surgical intervention. International Study of Unruptured Intracranial Aneurysms investigators. N Engl J Med 1998;339:1725-33.

14. Raaymakers TW, Rinkel GJ, Limburg M, et al. Mortality and morbidity of surgery for unruptured intracranial aneurysms: a meta-analysis. Stroke 1998;29:1531-8.

15. Raymond J. Managing unruptured aneurysms: the ethical solution to the dilemma. Can J Neurol Sci 2009;36:138-42.

16. Wiebers DO, Whisnant JP, Huston J III, et al. Unruptured intracranial aneurysms: natural history, clinical outcome, and risks of surgical and endovascular treatment. Lancet 2003;362:103-10.

17. Higgins J, Green S. Cochrane handbook for systematic reviews of interventions http://www.cochrane-handbook.org (accessed 10 Apr 2011).

18. Stroup DF, Berlin JA, Morton SC, et al. Meta-analysis of observational studies in epidemiology: a proposal for reporting. Meta-analysis of observational studies in epidemiology (MOOSE) group. JAMA 2000;283:2008-12.

19. Moher D, Liberati A, Tetzlaff J, et al. Preferred reporting items for systematic reviews and meta-analyses: the PRISMA statement. BMJ 2009;339:b2535.

20. Jennett $\mathbf{B}$, Bond $\mathrm{M}$. Assessment of outcome after severe brain damage. Lancet 1975;1:480-4.

21. Rankin J. Cerebral vascular accidents in patients over the age of 60. ii. Prognosis Scott Med J 1957;2:200-15.

22. Botterell EH, Lougheed WM, Scott JW, et al. Hypothermia, and interruption of carotid, or carotid and vertebral circulation, in the surgical management of intracranial aneurysms. J Neurosurg 1956;13:1-42. 
23. Brinjikji W, Rabinstein AA, Nasr DM, et al. Better outcomes with treatment by coiling relative to clipping of unruptured intracranial aneurysms in the United States, 2001-2008. AJNR Am J Neuroradiol 2011;32:1071-5.

24. Vandenbroucke JP, von Elm E, Altman DG, et al. Strengthening the reporting of observational studies in epidemiology (STROBE): explanation and elaboration. Epidemiology 2007; 18:805-35

25. von Elm $\mathrm{E}$, Altman DG, Egger $\mathrm{M}$, et al. The strengthening the reporting of observational studies in epidemiology (STROBE) statement: guidelines for reporting observational studies. Lancet 2007;370:1453-7.

26. Higgins JP, Thompson SG. Quantifying heterogeneity in a meta-analysis. Stat Med 2002;21:1539-58.

27. Higgins JP, Thompson SG, Deeks JJ, et al. Measuring inconsistency in meta-analyses. BMJ 2003;327:557-60.

28. Egger M, Davey Smith G, Schneider M, et al. Bias in meta-analysis detected by a simple, graphical test. BMJ 1997;315:629-34.

29. Souza JP, Pileggi C, Cecatti JG. Assessment of funnel plot asymmetry and publication bias in reproductive health meta-analyses: an analytic survey. Reprod Health 2007;4:3.

30. Sterne JA, Egger M. Funnel plots for detecting bias in meta-analysis: guidelines on choice of axis. J Clin Epidemio 2001;54:1046-55.

31. Hindman BJ, Todd MM, Gelb AW, et al. Mild hypothermia as a protective therapy during intracranial aneurysm surgery: a randomized prospective pilot trial. Neurosurgery 1999;44:23-32.

32. Lozier AP, Kim GH, Sciacca RR, et al. Microsurgical treatment of basilar apex aneurysms: perioperative and long-term clinical outcome. Neurosurgery 2004:54:286-96

33. Steinberg GK, Drake CG, Peerless SJ. Deliberate basilar or vertebral artery occlusion in the treatment of intracranial aneurysms. Immediate results and long-term outcome in 201 patients. J Neurosurg 1993;79:161-73.
34. Hauck EF, Wohlfeld B, Welch BG, et al. Clipping of very large or giant unruptured intracrania aneurysms in the anterior circulation: an outcome study. J Neurosurg 2008;109:1012-18.

35. Kim JE, Lim DJ, Hong CK, et al. Treatment of unruptured intracranial aneurysms in South Korea in 2006 : a nationwide multicenter survey from the Korean Society of Cerebrovascular Surgery. J Korean Neurosurg Soc 2010;47:112-18.

36. Yasunaga H, Matsuyama $Y$, Ohe K. Risk-adjusted analyses of the effects of hospita and surgeon volumes on postoperative complications and the modified Rankin scale after clipping of unruptured intracranial aneurysms in Japan. Neurol Med Chir (Tokyo) 2008; $48: 531-8$.

37. King JT Jr, Berlin JA, Flamm ES. Morbidity and mortality from elective surgery for asymptomatic, unruptured, intracranial aneurysms: a meta-analysis. J Neurosurg 1994;81:837-42.

38. Molyneux AJ, Kerr RS, Birks J, et al. Risk of recurrent subarachnoid haemorrhage, death, or dependence and standardised mortality ratios after clipping or coiling of an intracranial aneurysm in the International Subarachnoid Aneurysm Trial (ISAT): long-term follow-up. Lancet Neurol 2009;8:427-33.

39. Tsutsumi K, Ueki K, Usui M, et al. Risk of subarachnoid hemorrhage after surgical treatment of unruptured cerebral aneurysms. Stroke 1999;30:1181-4.

40. Qureshi Al, Suri MFK, Nasar A, et al. Trends in hospitalization and mortality for subarachnoid hemorrhage and unruptured aneurysms in the United States. Neurosurgery 2005;57:1-7.

41. Egger M, Zellweger-Zahner T, Schneider M, et al. Language bias in randomised controlled trials published in English and German. Lancet 1997:350:326-9.

42. Juni $\mathbf{P}$, Holenstein $F$, Sterne J, et al. Direction and impact of language bias in meta-analyses of controlled trials: empirical study. Int J Epidemiol 2002:31:115-23.

43. Raymond J, Darsaut TE, Molyneux AJ. A trial on unruptured intracranial aneurysms (the TEAM trial): results, lessons from a failure and the necessity for clinical care trials. Trials 2011;12:64. 